\title{
FIELD MEASUREMENT OF THE MAGNET PROTOTYPES FOR THE VSX PROJECT
}

\author{
T. Koseki ${ }^{1}$, Y. Kamiya, H. Kudo, N. Nakamura, T. Shibuya, \\ K. Shinoe, H. Takaki and Y. Takiyama, The Institute for Solid State Physics (ISSP), \\ The University of Tokyo, Tokyo, Japan \\ Y. Kobayashi, Photon Factory, High Energy Research Organization (KEK), Ibaraki, Japan \\ K. Kuno, Energy \& Industrial Research Center, Mitsubishi Electric Corporation, Kobe, Japan
}

\begin{abstract}
Prototypes of the dipole and the fast steering magnet for the VSX project have been fabricated and measured. The field mapping and the end-shim correction were carried out for the dipole, and the frequency response was tested up to $2 \mathrm{kHz}$ for the fast steering. The design of the magnets and measured results are presented.
\end{abstract}

\section{INTRODUCTION}

The University of Tokyo has been promoting a project to construct a VUV and Soft X-ray (SX) synchrotron radiation facility in a new campus of the university, Kashiwa campus. The project, which is called "VSX project", is composed of two phases. The first is to construct a $1.0 \mathrm{GeV}$ racetrack ring which has an emittance of $0.7 \mathrm{~nm} \cdot \mathrm{rad}$, a circumference of about $230 \mathrm{~m}$ and two 30 $\mathrm{m}$ long straight sections for insertion devices [1]. It can reach an emittance of diffraction limit and can provide extremely high-brilliance radiation more than $10^{20}$ [photons $/ \mathrm{sec} / \mathrm{mm}^{2} / \mathrm{mrad}^{2} / 0.1 \%$ b.w.] in the region between $100 \mathrm{eV}$ and $1 \mathrm{keV}$. The second is to construct a $2.0 \mathrm{GeV}$ ring, which has a circumference of $388 \mathrm{~m}$, an emittance of $5 \mathrm{~nm} \cdot \mathrm{rad}$ and 16 long straight sections [2]. High-brilliance synchrotron radiation over a wide range from VUV and SX can be generated by various kinds of insertion devices.

We have been carrying out the design study and R\&D's of the magnet system for both the $1.0 \mathrm{GeV}$ and $2.0 \mathrm{GeV}$ rings. Detailed design of main and corrector magnets for these rings has been almost completed. For the $1.0 \mathrm{GeV}$ ring, prototypes of the dipole, quadrupole and fast steering are being fabricated now. The quadrupole will be delivered to ISSP in this May and the dipole and steering in October. For the $2.0 \mathrm{GeV}$ ring, the dipole, quadrupole, sextupole and fast steering prototypes have been already fabricated by Mitsubishi Electric Corporation, and their field measurements are well under way at ISSP. Some results of the dipole, quadrupole and sextupole measurement have been reported in elsewhere [3,4].

In this paper, we present recent progress of the magnetic measurement of the dipole and the fast steering prototypes for the $2.0 \mathrm{GeV}$ ring. The measurement is being carried out using a computer-controlled 3D mapping system with a Hall probe unit (SERIES-9900 gaussmeter, F. W. BELL). For the dipole measurement, an NMR system (PT2025, METROLAB) is also used to obtain the absolute field strength and to calibrate the Hall probe unit.

\section{DIPOLE MAGNET}

Figure 1 shows the end view of the dipole prototype. The magnet core has C-type rectangular configuration and is made of forged low-carbon solid-steel. The shape of pole profile has been optimized using the 2D program LINDA to obtain a field uniformity better than $5 \times 10^{4}$ over a horizontal region of $\pm 40 \mathrm{~mm}$. The main parameters of the dipole are listed in Table 1.

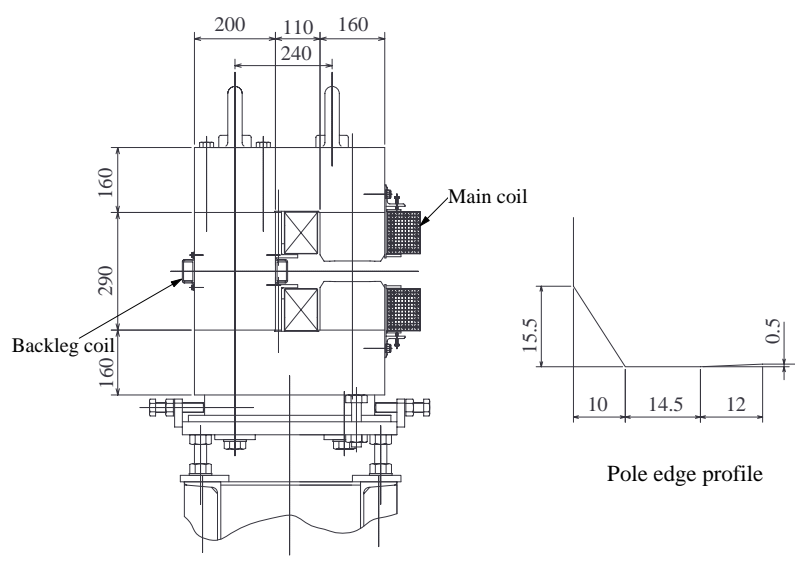

Figure 1: End view of the dipole prototype

Figure 2 shows the measured longitudinal field distribution along the design orbit. The excited field strength at the center of the magnet is $1.006 \mathrm{~T}$. The effective length, defined as the field integral along the design orbit divided by the field at the magnet center, was calculated to be $1364.0 \mathrm{~mm}$.

\footnotetext{
1 \# Email: koseki@issp.u-tokyo.ac.jp
} 
Table 1: The parameters of the dipole prototype

\begin{tabular}{lc}
\hline Bending angle & $11.25^{\circ}$ \\
Bending radius & $6.626 \mathrm{~m}$ \\
Gap height & $50 \mathrm{~mm}$ \\
Core length & $1.299 \mathrm{~m}$ \\
Maximum field strength & $1.26 \mathrm{~T}$ \\
Turns / pole & 30 \\
Maximum current & $960 \mathrm{~A}$ \\
Conductor size & $16 \times 15-\phi 9 \mathrm{~mm}$ \\
Resistance & $21 \mathrm{~m} \Omega$ \\
Water flow & $37.81 / \mathrm{min}$ \\
\hline
\end{tabular}

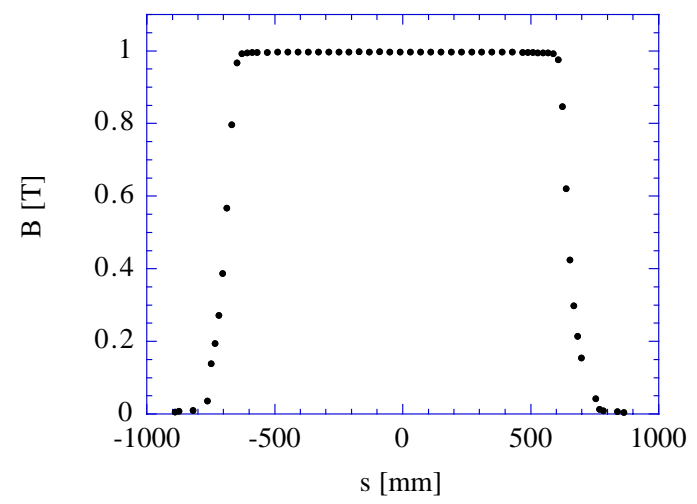

Figure 2: Longitudinal field distribution along the design orbit

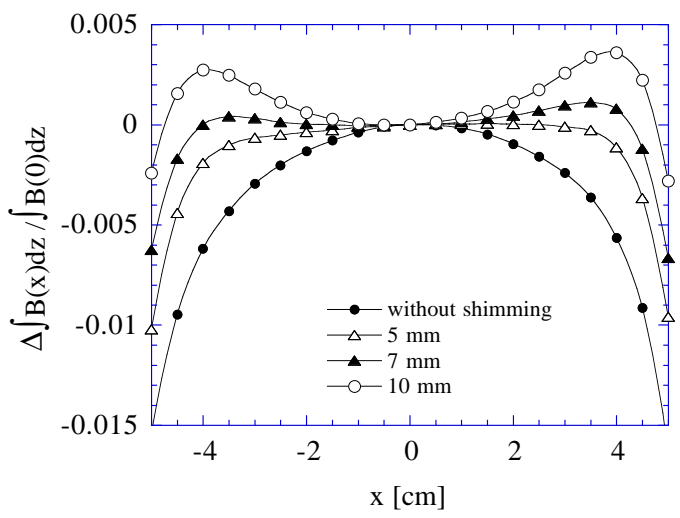

Figure 3: Integrated field profile

Near the magnet end, the sextupole component becomes relatively larger and it makes worse the horizontal field uniformity. In order to reduce the sextupole component, the end correction was carried out by shimming the magnet pole ends. The pieces of the end-shim are pureiron plates of $25 \mathrm{~mm} \times 139 \mathrm{~mm}$, and $2 \mathrm{~mm}$ or $5 \mathrm{~mm}$ thick. Figure 3 shows the integrated field uniformity for various thickness of end-shimming. Each integrated field is calculatedfrom mapping data along the longitudinal (zaxis) direction between $\mathrm{z}=450 \mathrm{~mm}$ and $\mathrm{z}=700 \mathrm{~mm}(\mathrm{z}=$ $650 \mathrm{~mm}$ is the position of the magnet end). This figure implies the most proper thickness is about $6 \mathrm{~mm}$, by which the integratedfield uniformity better than $5 \times 10^{-4}$ is obtained.

\section{FAST STEERING MAGNET}

The prototype of fast steering magnet is shown in Fig. 4. The steering has an aperture of $151 \mathrm{~mm}$ (width) $\times 46 \mathrm{~mm}$ (height) and provides both horizontal and vertical fields. The magnet core is composed of four glued stacks of 0.5 mm-thick silicon-steel lamination. The main parameters of the fast steering are listed in Table 2.

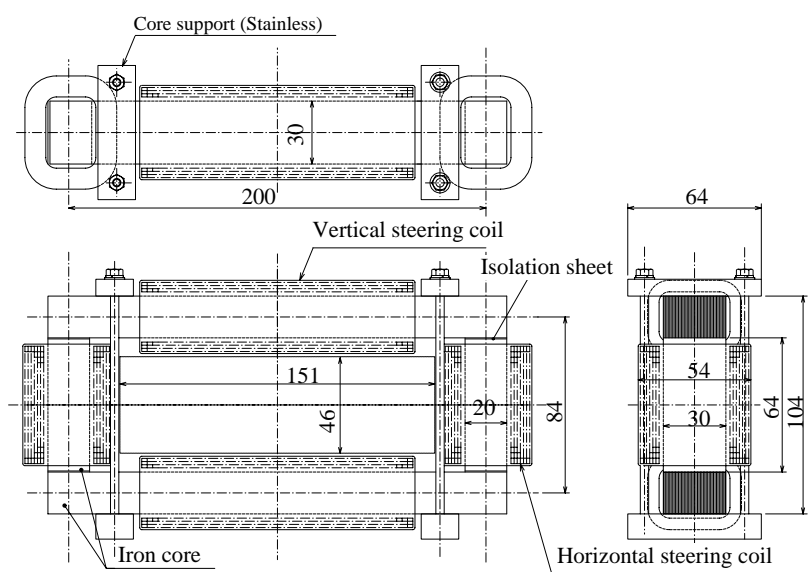

Figure 4: Fast steering prototype

Table 2: The parameters of the fast steering magnet

\begin{tabular}{ccc}
\hline & (Vertical & (Horizontal \\
& steering) & steering) \\
Deflection angle & $0.09 \mathrm{mrad}$ & $0.1 \mathrm{mrad}$ \\
Magnet gap & $180 \mathrm{~mm}$ & $64 \mathrm{~mm}$ \\
Turns / coil & 140 & 120 \\
Maximum current & $5 \mathrm{~A}$ & $5 \mathrm{~A}$ \\
Coil resistance & $0.25 \mathrm{~m} \Omega$ & $0.24 \mathrm{~m} \Omega$ \\
Coil inductance & $7 \mathrm{mH}$ & $8 \mathrm{mH}$ \\
Frequency range & $\mathrm{DC} \sim 100 \mathrm{~Hz}$ & $\mathrm{DC} \sim 100 \mathrm{~Hz}$ \\
\hline
\end{tabular}

The fast steering is expected to operate in a frequency range up to $100 \mathrm{~Hz}$. We measured the frequency response of the steering using a dynamic signal analyzer (HP35670A). A swept sine signal from the analyzer was fed into a bipolar AC power supply (IPM-BP series, IDX). The frequencyresponse of the output current of the power supply or the signal of the Hall probe was measured.

Figures 5 and 6 show the Bode diagram of the horizontal and vertical steerings, respectively. The data were measured with the output signal of the Hall probe 
put on the position of steering center. The output current of the power supply was set to be $800 \mathrm{~mA}$ peak-to-peak, that is a maximum current required for the fast orbit feedback system. A $1 \mathrm{~m}$ long vacuum chamber made of aluminum was inserted into the aperture of the steering magnet to examine the effect of eddy current induced on the chamber. The chamber has the same cross section as the beam duct of straight section of the VSX $2.0 \mathrm{GeV}$ ring [5]. As shown in these figures, the steering magnet itself has a good frequency response up to $1 \mathrm{kHz}$ for both the horizontal and vertical directions. Field attenuation due to the effect of eddy current on the vacuum chamber is not very serious in the frequency range up to $100 \mathrm{~Hz}$.

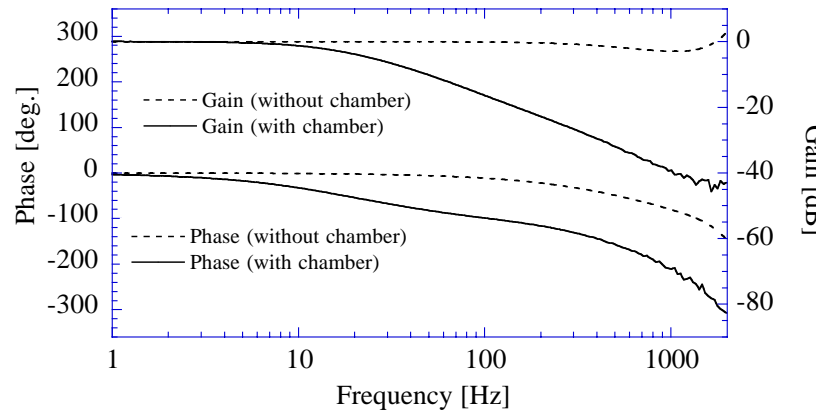

Figure 5: Frequency response of the horizontal steering field

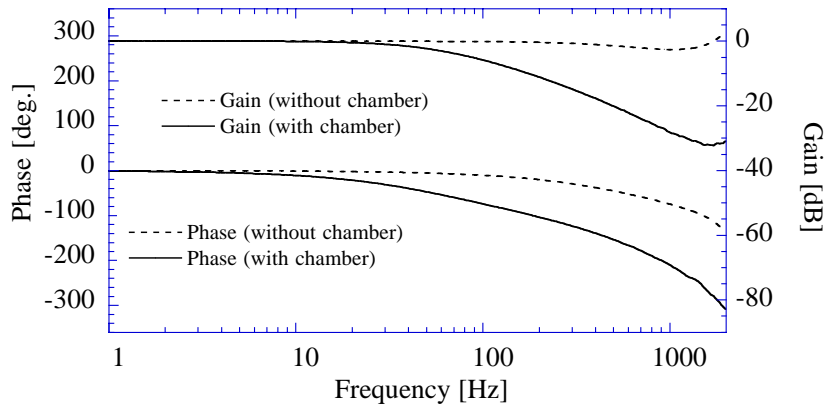

Figure 6: Frequency response of the vertical steering field

In the measurement of Figs. 5 and 6 , the data of the vertical steering was taken when the horizontal excitation switched off and vice versa. If both the steerings are excited in the same time, that is the actual situation of feedback operation, the interference between the two steerings may occur due to mutual inductance of the coils. Thus the frequencyresponse should be measured with both of them are switchedon. Two AC bipolar power supplies were used in the measurement and driven by the identical signal from the signal analyzer. Figure 7 shows the measuredBode diagram of the power supply output current for vertical steering when the horizontal steering switched on. The driving current was $800 \mathrm{~mA}$ peak-to-peak. The data without horizontal steering is also shown in this figure. There is no significant difference between them. It indicates that the magnetic field is given by a linear combination of the horizontal and vertical steering fields. In fact, even when both the steerings are switched on, the frequency response measured with the Hall probe well agrees with the data in Figs. 5 and 6. It means that independent feedback operation in the horizontal and vertical directions is possible using these steering magnets.

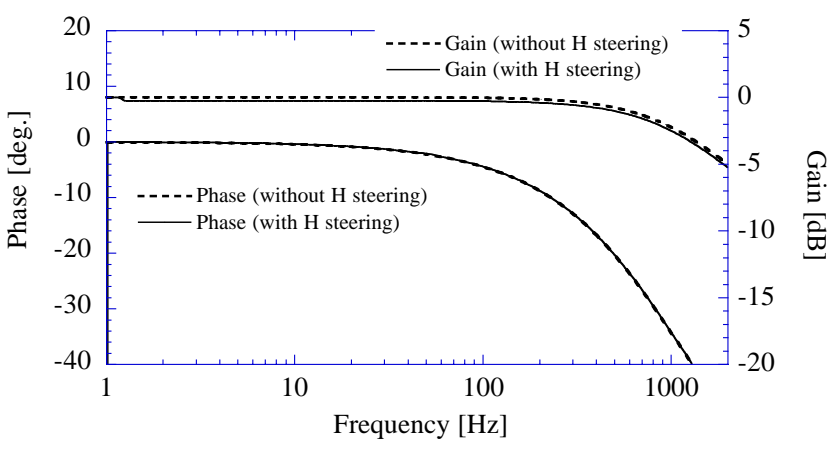

Figure 7: Frequency response of the output current monitor of the vertical steering

\section{REFERENCES}

[1] H. Takaki, K. Harada, T. Koseki, N. Nakamura, Y. Kamiya and Y. Kobayashi, "The Lattice of $1.0 \mathrm{GeV}$ VSX Storage Ring", in these proceedings.

[2] H. Takaki, T. Koseki, N. Nakamura, Y. Kamiya, Y. Kobayashi and T. Nagatsuka, "A Lattice for the Future Project of VUV and Soft X-ray High-Brilliant Light Source", 1997 PAC, Vancouver, (1997).

[3] T. Koseki, Y. Kobayashi, H. Takaki, N. Nakamura, Y. Kamiya and K. Kuno, "The Magnet of the VSX Light Source", APAC98, Tsukuba, (1998).

[4] T. Koseki, Y. Kamiya, H. Kudo, N. Nakamura, K. Shinoe, H. Takaki, Y. Takiyama, Y. Kobayashi and K. Kuno, "Magnetic Measurement of the VSX Prototype Magnets", EPAC98, Stockholm, (1998).

[5] N. Nakamura and T. Koseki, "Influence of Resistive Wall Impedance on the VSX Light Source", 1997 PAC, Vancouver, (1997). 